PS16.06.04 CRYSTAL GROWTH OF LITHIUM TRIBORATE FROM $\mathrm{Li}_{2} \mathrm{OB}_{2} \mathrm{O}_{3}$ SOLUTION. H.G. Kim, J. K. Kang, S.J. Chung, Dept. of Inorganic Materials Engineering, Seoul National Univ., Seoul 151-742, Korea

The non-linear optical characteristics of lithium triborate (abbreviated to LBO) are superior to those of KTP, BBO etc., especially in THG, OPO fields. But, the high viscosity of solution makes serious problems in crystal growth. In this study, $L B O$ single crystals were grown from $\mathrm{Li}_{2} \mathrm{O}-\mathrm{B}_{2} \mathrm{O}_{3}$ solutions. And the effects of seed orientations on crystal quality and morphology were investigated. The morphology of grown crystals was usually formed with large $\{110\}$ faces and small $\{011\},\{111\}$ faces.

The morphology of crystals grown from seeds parallel to $\langle 100\rangle$, $<001>$ direction were symmetrical. But, the seeds perpendicular to (110), (011) faces made the crystal morphology asymmetrical along growth axis.

Second phase was detected in some crystals grown under inadequate condition by $\mathrm{x}$-ray powder diffraction, The composition of inclusion was determined as $\mathrm{Li}_{3} \mathrm{~B}_{7} \mathrm{O}_{12}$ by heat treatment of $3 \mathrm{Li}_{2} \mathrm{O} .7 \mathrm{~B}_{2} \mathrm{O}_{3}$ and $2 \mathrm{Li}_{2} \mathrm{O} .5 \mathrm{~B}_{2} \mathrm{O}_{3}$ glasses.

For the determination of crystal defects, $\mathrm{x}$-ray topographic observations were carried out. The non-linear optical properties for SHG, THG and OPO applications of grown crystals were measured.

\section{PS16.06.05 CRYSTAL STRUCTURE AND DEFECTS IN} CZOCHRALSKI-GROWN LiCaAlF 6 . D. Klimm, K. Seiranian, P. Reiche, Institut für Kristallzuichtung, Rudower Chaussee 6, Geb. 18.46, D-12489 Berlin, Germany

Two colquiriite type fluorides, $\mathrm{Cr}^{3+}: \mathrm{LiCaAlF}_{6}$ and $\mathrm{Cr}$ $3+: \mathrm{LiSrAlF}_{6}$, were reported as new tunable laser hosts ( $\mathrm{Lin}+$ Keszler, Chem. Mater. 4 (1992), 645). The $\mathrm{Cr}^{3} \div$ substitutes Al 3+ ions isomorphic, and thus very high doping levels can be obtained. Moreover, the segregation coefficient $\mathrm{k}=\mathrm{c}_{\text {sol }} / \mathrm{c}_{\text {liqu }}$ for the lasing ion $\mathrm{Cr}^{3}+$ is close to 1 , resulting in a homogeneous $\mathrm{Cr}$ distribution along the length of the growing crystal. Accordingly, both substances are suspected to have considerable advantages over conventional high power dye lasers.

Many authors reported on the occurrence of scattering centers in the Czoch ralski ( $\mathrm{CZ}$ ) grown crystals, that reduce the lasing efficiency. Usually these particles are attributed to $\mathrm{CrO}_{x}$ resulting from the moisture contained in the ambient atmosphere reacting with the $\mathrm{CrF}_{3}$ dopant during crystal growth.

This work reports on the automatizised $\mathrm{CZ}$ growth of $\mathrm{Cr}^{3+}: \mathrm{LiCaAlF}_{6}$ single crystals. Crystal growth was performed with RF heating using $P$ t crucibles under flowing nitrogen atmosphere $\left(<10\right.$ ppm $\mathrm{H}_{2} \mathrm{O}$ ). The quality of the boules was characterized by light microscopy and by means of EDX and X-ray topography. As usual, the near surface region of the crystals turns out to be free from scattering centers. Scattering centers in the interior of the crystals are investigated by transmission electron microscopy and by positron annihilation, respectively, and are discussed in terms of extrinsic $\left(\mathrm{CrO}_{\mathrm{X}}\right)$ as well as intrinsic defects. The relation between the ideal structure and the defect structure of the colquiriite type crystals is discussed.

This work was sponsored by BMBF (contract 624-4013-13N6750/2).
PS16.06.06 MELT GROWTH AND CHARACTERIZATION OF 2-METHYL-4-NITROANILINE CRYSTALS. Masaru Tachibanaa, Masaaki Satob, Kenichi Kojimaa, aFaculty of Science and bGraduate School of Integrated Science, Yokohama City University, 22-2 Seto, Kanazawa-ku, Yokohama 236, Japan

Recently considerable interest has developed for organic materials with extremely high optical nonlinearities. In particular, 2-methyl-4-nitroaniline (MNA) is one of the most promising materials for nonlinear optical devices. The growth of single crystals with large size and high quality is important for accurate characterization and technical applications of the materials. In this work, MNA single crystals were grown from melt by Bridgman method, and their perfection was examined by X-ray topography.

MNA powder was well-purified not only by sublimation at $90^{\circ} \mathrm{C}$ but also by zone-refining, where its melting point is $132^{\circ} \mathrm{C}$. The purified powder was deposited in a pyrex tube. The tube was placed in a vertical Bridgman furnace with two temperature zones, where the temperatures of upper zone and lower zone were kept at $139^{\circ} \mathrm{C}$ and at $110^{\circ} \mathrm{C}$, respectively. The material in the tube was completely molten at the upper zone. The molten material was crystallized at the lower zone by slowly pulling down the tube at the rate of $0.8 \mathrm{~mm} / \mathrm{h}$. After the crystallization, the crystal was cooled down to room temperature at $0.5^{\circ} \mathrm{C} / \mathrm{h}$. Consequently single crystals with cylindrical shapes of $5 \mathrm{~mm}$ diameters were grown in the tubes. Large volumes of the crystals were optically clear and appeared pale yellow. The perfection of the grown crystals was examined not only by projection topography but also section topography. As a result, the crystal defects, especially dislocations, were characterized.

In addition, the nonlinear optical properties of the grown MNA crystals were investigated by the measurements of second harmonic generation $(\mathrm{SHG})$

PR16.06.07 THE METAL MELT STATE EHFECT ON THE MONOCRYSTAL GROWTH. N.M. Kochegura, Institute of Foundry Problems, Ukrainian National Academy of Sciences, Kiev, Ukraine.

The work is devoted to the study of the influence of the state of high temperature resistant nickel melt on the monocrystal growth in the compound Ni-Cr-Mo-Ti-Al-Co-Nb-C system. The monocrystal structure was formed in directionally solidified process by Bridgeman-Stockbarger's method. Monocrystal specimens were grown from ordinary or homogenised at $2000-2020 \mathrm{~K}$ melts. The monocrystals had a dendritic branch which was grown from crystal nuclear-seeding along the direction $<001>$. The specimens which was grown from the ordinary melt had a dendrite structure with different irregular intervals between branches. There were "mat spot" on periphery. This "spots " had structure with small equalaxis crystal. The specimens grown from homogenised melts had structure $<001>$ without psevdograin. In this case dendrite cross-section had the regular form. The dendrite axis' and iteraxis space didn't differ neither in size nor in the configuration at the centre as well as at the periphery of the specimens.

These results may be explained thus the homogenised melt creates favourable conditions for the monocrystal growth mostly due to the decrease the deactivation of crystal nucleuses and cluster decays of such metals as W, Mo, Nb, etc. 